\title{
Menstrual hygiene: an important prognosticator of excessive vaginal bleeding in the high focused states of India
}

\begin{abstract}
Background: There is increasing evidence of challenges that girls and women face in managing menstruation and vaginal bleeding. In many societies, these are handled behind the scenes as cultural taboos.

Objectives: The study aims to address the effects of menstrual hygiene on excessive vaginal bleeding during and after delivery.

Methods: The study used data from the fourth round of the National Family Health Survey. The study included 146,121 women aged 15-24 years from high focused states of India. Chi-Square analysis was used to examine the significance of menstrual hygiene on excessive vaginal bleeding during and after delivery. In addition, binary logistic regression was used for the assessment of the adjusted effects.

Results: The prevalence of excessive vaginal bleeding during and after delivery ranged from 4.3 percent to 12.8 percent and 2.6 percent to 8.2 percent, respectively. Women who use hygienic method of protection to prevent blood stains during menstrual period were at lower risk of suffering from excessive vaginal bleeding during $(\mathrm{OR}=0.80 ; 95 \% \mathrm{CI}=[0.77$, $0.84])$ and after delivery $(\mathrm{OR}=0.91 ; 95 \% \mathrm{CI}=[0.86,0.97])$. Women who had terminated pregnancy, having higher BMI and lower level of education were also at higher risk.
\end{abstract}

Conclusion: The study portrays evidence of a strong association between menstrua hygiene and excessive vaginal bleeding during and after delivery. Therefore, attention in research, practice and policy, including improved education, training and communication, is necessary to break the silence around girls and women's regarding menstrual hygiene.

Keywords: menstrual hygiene, delivery, vaginal bleeding, high focused states, India, menstrual hygiene: an important prognosticator of excessive vaginal bleeding in the high focused states of India
Volume 7 Issue 6 - 2021

\author{
Wahengbam Bigyananda Meitei,' Aditi² \\ 'PhD Scholar Biostatistics \& Demography, International Institute \\ for Population Sciences, India \\ 2PhD Scholar Population Studies, International Institute for \\ Population Sciences, India
}

\begin{abstract}
Correspondence: Aditi, PhD Scholar Population Studies, International Institute for Population Sciences, Mumbai-400088, Email aditi.chaudhary72@gmail.com
\end{abstract}

Received: December 07, 2021 | Published: December 27, 2021

\section{Introduction}

Menstruation, or the monthly shedding of the uterine lining, is the most significant part of a woman's menstrual cycle. The first menstruation (menarche) occurs at a very tender age, between 11 to 15 years. ${ }^{1}$ The onset of menstruation is one of the most substantial changes that occur in adolescent girls. On any day, over 800 million adolescent girls and women worldwide are menstruating. ${ }^{2}$ However, the complications and challenges that surround it are understudied, especially in the resource-poor scenario.

Although menstruation is a natural process, it is widely linked to several misconceptions and practices. There is increasing evidence of challenges upon girls and women in managing menstruation and vaginal bleeding. ${ }^{2,3}$ It is widely regarded as unclean or dirty and is handled behind the scenes as cultural taboos. ${ }^{4}$ This vulnerability of women holds particularly in India, where female children are neglected. ${ }^{1,5}$ Isolation of the menstruating girls and their restrictions on them have underpinned a negative attitude towards this natural process. With insufficient information, ongoing social, cultural and hygiene taboos, inadequate sanitation and waste disposal facilities, menstruating girls or women experience fear, shame and confusion while facing the challenges in managing their menstrual hygiene. ${ }^{6-8}$ Maintaining good hygiene with challenges like access to water, restrooms, and sanitary products is poor, and where the discussions regarding menstruation is considered, a taboo is very difficult. These WASH inadequacies directly affect women's health and put them at serious health risk. ${ }^{2,7}$ There has also been evidence of how inadequate privacy, sanitation facilities and disposal systems make women vulnerable to mental, emotional and physical problem during their cycle days. ${ }^{9}$ Based on the information about the challenges that the women in the low and middle-income countries are facing regarding the management of menstrual hygiene, some researchers are even hypothesizing that the evidence regarding poor menstrual hygiene that they have, represents the tip of the iceberg. ${ }^{2}$ In reality, the problem is much severe.

A variety of factors affect menstrual behaviours, the most important being education, economic and residential status. Researchers have been discussing how the onset of menstruation also meant the onset of new restrictions on the movement of girls. ${ }^{5,9}$ They found evidence of girls' abilities to pursue an education or a career they wanted to pursue hindered by menstruation. Poverty is another factor that prevents women from managing their hygiene effectively; there is evidence of women not using commercially sold sanitary napkins since there is a cost associated with it. The women's body mass index (BMI) also affects the amount of blood flow of women. Obese women usually have irregular cycles and are also prone to quite heavy menstrual flow during their periods. ${ }^{10,11}$ 
Women's proper hygiene during menstruation is quite important, as it increases vulnerability to reproductive tract infections (RTI) and other health risks. It is the cause of various morbidities faced by women in later stages of life. Maternal morbidity is a precursor to maternal mortality and a potential cause of lifetime disability and poor quality of life. ${ }^{12}$ Excessive vaginal bleeding during and after delivery can cause a severe drop in women's blood pressure and may lead to shock and even death if not treated appropriately. ${ }^{13}$ Haemorrhage is one of the top three direct obstetric causes of maternal mortality worldwide, with most deaths occurring within 24-48 hours of delivery. ${ }^{14}$

The interplay of socio-economic status, menstrual hygiene practices and diseases related to the urinary tract are noticeable. Today millions of women are sufferers of RTI and its complications, and in various cases, the infection transmits to the offspring of the pregnant mother. ${ }^{1}$ Globally, 289,000 maternal deaths were estimated in 2013, a decline of 45 percent from 1990, but 300 million women suffer from short-term or long-term morbidities due to pregnancy or childbirth. ${ }^{15,16}$ The Maternal Mortality Ratio in India during 200406 was 254 per 100,000 live births with wide geographic variations, which slightly declined to 212 per 100,000 live births in 2007-09. ${ }^{17}$ In 2014-16 the Maternal Mortality Ratio was 130 per 100,000 live births. ${ }^{18}$ The government of India is trying hard to bring down the level of maternal mortality and morbidity. The recently introduced National Health Mission focused on the process and developed the outcome indicators to reflect equity, quality, efficiency and responsiveness. The Mission also aimed to reduce the maternal mortality ratio to 1 per 1000 live births. ${ }^{19}$ However, it is still a vexing problem. The maternal mortality ratio in the "high focused states" is relatively higher than in other states of the country. It was estimated at 188 per 100,000 live births. ${ }^{18}$ Maternal health care utilization is also relatively low in these states. ${ }^{20,21}$ The number of women facing maternal morbidities and hence mortality is enormous in these states. Therefore, it would be worthwhile to know what are the factors that underplay a significant role here. Thus, keeping in mind the perceived quality of care and the challenges faced by the girls or women in these states, the study aims to assess the effects of menstrual hygiene on excessive vaginal bleeding during and after delivery in the high focused states of India.

\section{Methods and materials}

Data from the fourth round of the National Family Health Survey was used for the analysis. The National Family Survey is a nationally representative survey conducted across all states and union territories of India. In its fourth round, it collected information on fertility, infant and child mortality, the practice of family planning, maternal and child health, reproductive health, nutrition, anaemia, utilization and quality of health and family planning services and HIV/AIDS. Since our study is primarily focused on women's reproductive health, we extensively used the women file of the survey for our analysis. The study focused on the nine highly focused states of India viz. Assam, Bihar, Chhattisgarh, Jharkhand, Madhya Pradesh, Odisha, Rajasthan, Uttar Pradesh and Uttarakhand. These states are grouped under "high focused states" due to their poor maternal and child health outcome indicators and high fertility rates. These states account for about $48.5 \%$ of India's total population. The study included 146,121 women aged 15-24 years.

One of the maternal morbidities, excessive vaginal bleeding during delivery and within two months after delivery, has been considered the outcome variable. The prognosticator of the outcome variable, menstrual hygiene, has been categorized as hygienic and unhygienic. Those women using locally prepared napkins, sanitary napkins and tampons during their menstrual days have been categorized as hygienic. And those women who are using cloths and other material or not using are categorized as unhygienic. Other than menstrual hygiene, we have also considered following socioeconomic and demographic characteristics viz. whether the women had terminated pregnancy (yes or no), body mass index of the women (less than 18.5, 18.5 to 24.9 and $24.9 \&$ above), level of education (no education, primary, secondary and higher), household wealth quintile (poorest, poorer, middle, richer and richest) and place of residence (rural and urban). Chi-Square analysis was used to examine the significance of menstrual hygiene on excessive vaginal bleeding during and after delivery. And binary logistic regression was used for the assessment of the adjusted effects. STATA 13 was used for executing the analysis.

\section{Results}

Figure 1 presents the prevalence of excessive vaginal bleeding during and after delivery in high focus states of India. In all states, the prevalence of excessive vaginal bleeding is higher during delivery than after delivery. The prevalence of excessive vaginal bleeding during delivery in the high focus states ranged from 4.3 percent in Uttarakhand to 12.8 percent in Madhya Pradesh. While the prevalence of excessive vaginal delivery after delivery ranged from 2.6 percent in Uttarakhand to 8.2 percent in Bihar. States like Madhya Pradesh, Bihar, Odisha, Chhattisgarh, Jharkhand, Rajasthan and Assam have a relatively higher prevalence of excessive vaginal bleeding during delivery as compared to other states. Figure 1 also provides information on the significance of the estimates of the prevalence of excessive vaginal bleeding in the form of error bars. The narrow line of the error bar represents that the variation of the estimated prevalence of each state is not too large and reliable. It also shows that the estimated prevalence differs significantly from one state to another (Figure 1).

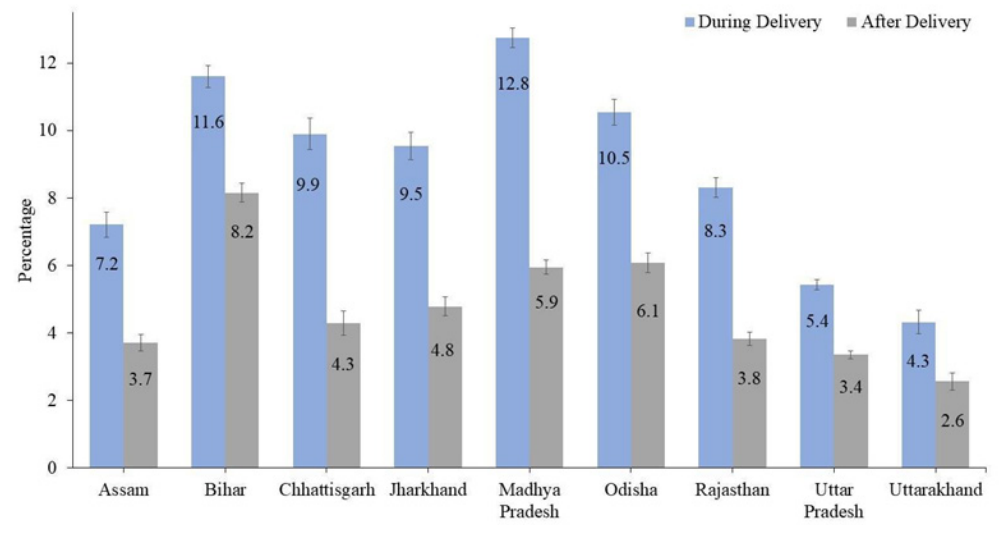

Figure I Prevalence of excessive vaginal bleeding during and after delivery in high focus states of India. 
Table 1 presents the prevalence of excessive vaginal bleeding during and after delivery by different socioeconomic and demographic characteristics. The prevalence of excessive vaginal bleeding during and after delivery among those women who were unhygienic during their menstrual period is higher than those who were hygienic. Its prevalence among unhygienic women were 10.46 percent $(95 \%$ $\mathrm{CI}=[10.25,10.67])$ and 5.49 percent $(95 \% \mathrm{CI}=[5.33,5.65])$ respectively. Compared to women who had no terminated pregnancy, the prevalence of excessive vaginal bleeding was higher among those women who had prior terminated their pregnancy. Its prevalence was 21.13 percent $(95 \% \mathrm{CI}=[20.18,22.07])$ during delivery and 12.20 percent $(95 \% \mathrm{CI}=[11.44,12.96])$ after delivery. With the increase in the women's body mass index, the prevalence of excessive vaginal bleeding also increased. On the contrary, as the women's education and socioeconomic status increases, the prevalence of excessive vaginal bleeding decreases. Its prevalence among women who had attained no formal education is 15.97 percent $(95 \% \mathrm{CI}=[15.47$, $16.47])$ during delivery and 8.79 percent $(95 \% \mathrm{CI}=[8.84,9.17])$ after delivery. While its prevalence among women who had attained higher education is 4.66 percent $(95 \% \mathrm{CI}=[4.35,4.97])$ during delivery and 2.82 percent $(95 \% \mathrm{CI}=[2.57,3.06])$ after delivery. Compared to women living in urban areas, the prevalence of excessive vaginal bleeding is higher among those living in rural areas. Its prevalence in rural areas are 9.37 percent $(95 \% \mathrm{CI}=[9.19,9.54])$ during delivery and 5.11 percent $(95 \% \mathrm{CI}=[4.98,5.24])$ after delivery.

Table I Prevalence of excessive vaginal bleeding before and after delivery by different socioeconomic and demographic characteristics

\begin{tabular}{|c|c|c|c|c|c|c|c|c|}
\hline \multirow{3}{*}{ Menstrual hygien } & \multicolumn{4}{|c|}{ During delivery } & \multicolumn{4}{|c|}{ After delivery } \\
\hline & \multirow[t]{2}{*}{ Prevalence } & \multicolumn{2}{|l|}{$95 \% \mathrm{Cl}$} & \multirow[t]{2}{*}{ p-value } & \multirow[t]{2}{*}{ Prevalence } & \multicolumn{2}{|l|}{$95 \% \mathrm{Cl}$} & \multirow[t]{2}{*}{ p-value } \\
\hline & & & & & & & & \\
\hline Unhygienic & 10.46 & 10.25 & 10.67 & $<0.01$ & 5.49 & 5.33 & 5.65 & $<0.01$ \\
\hline Hygienic & 6.43 & 6.24 & 6.61 & & 3.63 & 3.49 & 3.78 & \\
\hline \multicolumn{9}{|c|}{ Terminated pregnancy } \\
\hline No & 7.99 & 7.84 & 8.13 & $<0.01$ & 4.26 & 4.15 & 4.36 & $<0.01$ \\
\hline Yes & 21.13 & 20.18 & 22.07 & & 12.20 & 11.44 & 12.96 & \\
\hline \multicolumn{9}{|c|}{ Body mass index } \\
\hline Less than 18.5 & 7.81 & 7.58 & 8.04 & & 4.10 & 3.93 & 4.27 & $<0.01$ \\
\hline 18.5 to 24.9 & 9.04 & 8.85 & 9.23 & $<0.01$ & 4.90 & 4.76 & 5.05 & \\
\hline $24.9 \&$ above & 9.46 & 8.87 & 10.05 & & 5.33 & 4.88 & 5.79 & \\
\hline \multicolumn{9}{|c|}{ Level of education } \\
\hline No education & 15.97 & 15.47 & 16.47 & & 8.79 & 8.84 & 9.17 & \\
\hline Primary & 12.94 & 12.42 & 13.47 & $<0.01$ & 6.73 & 6.34 & 7.13 & $<0.01$ \\
\hline Secondary & 7.02 & 6.86 & 7.19 & & 3.72 & 3.60 & 3.84 & \\
\hline Higher & 4.66 & 4.35 & 4.97 & & 2.82 & 2.57 & 3.06 & \\
\hline \multicolumn{9}{|l|}{ Wealth quintile } \\
\hline Poorest & 10.62 & 10.32 & 10.92 & & 5.79 & 5.56 & 6.02 & \\
\hline Poorer & 9.21 & 8.91 & 9.50 & & 5.03 & 4.81 & 5.26 & \\
\hline Middle & 8.35 & 8.03 & 8.68 & $<0.01$ & 4.54 & 4.30 & 4.79 & $<0.01$ \\
\hline Richer & 7.20 & 6.86 & 7.55 & & 3.66 & 3.41 & 3.91 & \\
\hline Richest & 5.19 & 4.86 & 5.51 & & 2.68 & 2.45 & 2.91 & \\
\hline \multicolumn{9}{|c|}{ Place of residence } \\
\hline Urban & 6.23 & 5.97 & 6.48 & $<0.01$ & 3.15 & 2.96 & 3.33 & $<0.01$ \\
\hline Rural & 9.37 & 9.19 & 9.54 & & 5.11 & 4.98 & 5.24 & \\
\hline
\end{tabular}

Note: $\mathrm{Cl}=$ Confidence Interval; $\mathrm{p}$-value refers to Chi-Square $\mathrm{p}$-value

Table 2 presents the effects of menstrual hygiene on excessive vaginal bleeding during and after delivery adjusted for different demographic and socioeconomic characteristics. With references to unhygienic practices during menstrual period, women who adopt hygienic practices are less likely to suffer from excessive bleeding during $(\mathrm{OR}=0.80 ; 95 \% \mathrm{CI}=[0.77,0.84])$ and after $(\mathrm{OR}=0.91 ; 95 \%$
$\mathrm{CI}=[0.86,0.97])$ delivery. Women who had terminated pregnancy are more likely to suffer from excessive bleeding both, during (OR $=2.67 ; 5 \% \mathrm{CI}=[2.51,2.84])$ and $\operatorname{after}(\mathrm{OR}=2.70 ; 95 \% \mathrm{CI}=[2.50$, 2.92]) delivery. As the body mass index of the women increases, the risk of suffering from excessive vaginal bleeding both during and after delivery is likely to increase. On the contrary, the risk of suffering 
from excessive vaginal bleeding decreases as education increases. Compared to women in the household with poorest socioeconomic status, women in the household with middle $(\mathrm{OR}=1.08 ; 95 \%$ $\mathrm{CI}=[1.02,1.14])$ and richer $(\mathrm{OR}=1.08 ; 95 \% \mathrm{CI}=[1.01,1.15])$ socioeconomic status are more likely to suffer from excessive vaginal bleeding during delivery. However, women in households with the richest socioeconomic status are less likely to suffer from excessive vaginal bleeding after delivery $(\mathrm{OR}=0.82 ; 95 \% \mathrm{CI}=[0.73,0.93])$. A compared to women living in urban areas, women living in rural areas are more likely to suffer from excessive vaginal bleeding during (OR $=1.31 ; 95 \% \mathrm{CI}=[1.24,1.38])$ and $\operatorname{after}(\mathrm{OR}=1.39 ; 95 \% \mathrm{CI}=[1.29$, 1.50]) delivery (Table 2).

\section{Discussion}

The study discusses the effects of menstrual hygiene on maternal morbidity, particularly excessive vaginal bleeding during and after delivery. It unravels some of the hidden facts of the relationship between the duo in the high-focused states of India.

The "high focused states" are the states where the maternal health outcomes are poor and fertility rates are high. ${ }^{22,23}$ Despite continuous efforts from policymakers to improve the country's maternal conditions, particularly for "high focused states". Compared to other Indian states, the maternal conditions in these states are still considerably poor with wide variations. ${ }^{24}$ Similarly, the study shows a wide variation in the prevalence of excessive vaginal bleeding during and after delivery between these states. However, within states, estimates are significantly different and reliable. A state like Uttarakhand has the lowest prevalence of excessive vaginal bleeding both during and after delivery. Whereas Madhya Pradesh and Bihar respectively have the highest prevalence of excessive vaginal bleeding during and after delivery. Several authors have opined that emphasis on menstrual hygiene should be strengthened. ${ }^{2}$ Managing menstrual hygiene is fundamental to reducing the burden of maternal morbidity, a precursor of maternal mortality.,12 Both bivariate and multivariate analysis show a significant association between menstrual hygiene and maternal morbidities, i.e., excessive vaginal bleeding during and after delivery. Compared to women who prefer to use the unhygienic method, women who prefer to use the hygienic method for managing menstrual bleeding are less likely to suffer from maternal morbidities. Reproductive tract infections and urinary tract infections, which have become epidemics that devastate women's health, is closely interrelated with poor menstrual hygiene. Therefore, proper menstrual hygiene and correct knowledge and beliefs can protect womenfolk from this kind of suffering. Various studies have stressed the importance of females being aware of menstrual hygiene and its linkages with better maternal health. ${ }^{1,5}$

Women's ability to manage menstrual hygiene appropriately involves various social, cultural and financial constraints. These constraints negatively affect their ability to manage menstrual hygiene leading to major maternal health risks. ${ }^{4}$ Limited availability of clean, accessible water and sanitation facilities adds to the existing problem in managing menstrual hygiene. ${ }^{4}$ Results show a significant relationship between higher BMI and the amount of blood flow. Women who have higher BMI are more likely to have an excessive amount of blood flow. The findings go in line with another study conducted in Port-Said city. ${ }^{11}$ Excessive blood loss can also lead to anaemia and other complications. Obesity (BMI $>=24.9)$ is one of the underlying predisposing factors for excessive blood loss. ${ }^{10}$

The study shows that the risk of excessive vaginal bleeding decreases as the level of education and household socioeconomic status increases. Other studies have also highlighted the importance of educational intervention leading to significant changes in girls' knowledge regarding managing their menstrual hygiene..$^{5,8,23}$ There are various studies that are consistent with the finding that socioeconomic status is an inhibitor to practising safe menstrual hygiene, poor women use old cloth during their periods because sanitary pads involve a cost. The use of cloth has been linked to increased chances of microbial growth and hence infections. ${ }^{5,7}$ Not only this, they wash and reuse the same cloth, which at times can be really unhygienic because of not being properly washed or the cloth not being dried properly. ${ }^{1,25}$ The study also showed that the prevalence of excessive vaginal bleeding is higher among women residing in rural areas than women in urban areas. A higher level of awareness regarding menstrual hygiene among urban adolescent girls compared to rural has been found in other studies. ${ }^{1,5,25}$ The above findings reinforce the urgent need to encourage safe and hygienic practices among women and dispel the taboos, misconceptions and restrictions regarding menstruation. Women's lack of meaningful participation against the cultural taboos and practices surrounding menstruation hinders implementing practical solutions. This requires longer-term strategies to bring about cultural change.

The study shows a significant association between menstrual hygiene and maternal morbidities. However, the association established in the study is an outcome from cross-sectional data. Its association could have been more reliable if we have considered longitudinal data for the analysis. But, data on maternal morbidity based on the longitudinal study is not available for these high focused states. In addition, the cases of excessive vaginal bleeding during and after delivery is self-reported. And, since the National Family Health Survey provide information on menstrual hygiene only for women whose age is between 15 years to 24 years, we are compelled to restrict our study to this age group only.

\section{Conclusion}

The study portrays evidence of a strong association between menstrual hygiene and excessive vaginal bleeding during and after delivery. While the risk of acquiring excessive vaginal bleeding decreases as the level of education of the women and socioeconomic status of the household increases. Therefore, attention in research, practice and policy, including improved education, training and communication, is necessary to break the silence around girls and women regarding menstrual hygiene. A key priority for society should be to let the girls and the women have the required knowledge, facilities and the cultural environment to manage menstruation hygienically and with dignity.

\section{Acknowledgments}

WBM conceptualized, designed the study and led the data analysis. WBM \& A wrote the first draft and contributed to the analysis, interpretation of the results, and writing. WBM provided overall supervision to the study. All authors approved the final submission of the study.

\section{Conflicts of interest}

The authors have no conflicts of interest relevant to this article to disclose.

\section{Funding}

The authors does not receive any financial support from any organization for the study. 


\section{References}

1. Dasgupta A, Sarkar M. Menstrual hygiene:how hygienic is the adolescent girl? Indian journal of community medicine: official publication of Indian Association of Preventive \& Social Medicine. 2008;33(2):77.

2. Sommer M, Caruso BA, Sahin M, et al. A time for global action:addressing girls' menstrual hygiene management needs in schools. PLoS medicine. 2016;13(2).

3. Sommer M, Hirsch JS, Nathanson C, et al. Comfortably, safely, and without shame:defining menstrual hygiene management as a public health issue. American Journal of Public Health. 2015;105(7):13021311.

4. Sommer M, Phillips-Howard PA, Mahon T, et al. Beyond menstrual hygiene:addressing vaginal bleeding throughout the life course in low and middle-income countries. BMJ global health. 2017;2(2):e00405.

5. Thakre SB, Thakre SS, Reddy M, et al. Menstrual hygiene:knowledge and practice among adolescent school girls of Saoner, Nagpur district. $J$ Clin Diagn Res. 2011;5(5):1027-1033.

6. Mahon T, Fernandes M. Menstrual hygiene in South Asia: a neglected issue for WASH (water, sanitation and hygiene) programmes. Gender \& Development. 2010;18(1):99-113.

7. McMahon SA, Winch PJ, Caruso BA, et al. The girl with her period is the one to hang her head'Reflections on menstrual management among schoolgirls in rural Kenya. BMC international health and human rights. 2011;11(1):7.

8. Montgomery P, Ryus CR, Dolan CS, et al. Sanitary pad interventions for girls' education in Ghana: a pilot study. PloS one. 2012;7(10).

9. Sudeshna R, Aparajita D. Determinants of menstrual hygiene among adolescent girls: a multivariate analysis. Natl J Community Med. 2012;3(2):294-301.

10. Nouri M, Tavakkolian A, Mousavi SR. Association of dysfunctional uterine bleeding with high body mass index and obesity as a main predisposing factor. Diabetes \& Metabolic Syndrome:Clinical Research \& Reviews. 2014;8(1):1-2.

11. Abdella N, Abd-Elhalim EH, Attia AM. The Body Mass Index and Menstrual Problems among Adolescent Students. IOSR Journal of Nursing and Health Science (IOSR-JNHS). 2016;5(4):13-21.

12. Firoz T, Chou D, von Dadelszen P, et al. Measuring maternal health: focus on maternal morbidity. Bulletin of the World Health Organization. 2013;91:794-796.
13. Stanford Children's Health (SCH). Postartum Haemorrhage. 2019.

14. Hogan MC, Foreman KJ, Naghavi M, et al. Maternal mortality for 181 countries, 1980-2008:a systematic analysis of progress towards Millennium Development Goal 5. The lancet. 2010;375(9726):16091623 .

15. WHO U. UNFPA, World Bank. Maternal mortality in 2005:estimates developed by WHO, UNICEF.

16. World Health Organization. Trends in maternal mortality:1990 to 2013: estimates by WHO, UNICEF, UNFPA, The World Bank and the United Nations Population Division. 2014.

17. SRS. Special Bulletin on Maternal Mortality Ratio 2007-09. Office of the Registrar General of India. 2011.

18. SRS. Special Bulletin on Maternal Mortality Ratio 2014-16. Office of the Registrar General of India. 2018.

19. Ministry of Health and Family Welfare (MoHFW), Government of India. National Health Mission. 2020.

20. Singh PK, Kumar C, Rai RK, et al. Factors associated with maternal healthcare services utilization in nine high focus states in India: a multilevel analysis based on 14385 communities in 292 districts. Health policy and planning. 2014;29(5):542-559.

21. International Institute for Population Sciences (IIPS) and ICF. National Family Health Survey (NFHS-4), 2015-16:India. Mumbai: IIPS. 2017.

22. Mohanty SK, Srivastava A. Cost and utilisation of hospital based delivery care in empowered action group (EAG) states of India. Maternal and child health journal. 2013;17(8):1441-1451.

23. Das K, Ganguly N, Ghosh S. Factors Affecting Maternal Care Utilization in Empowered Action Group (EAG) States of India: Evidences from Annual Health Survey 2012-13. India J Indian Anthrop Soc. 2018;53:161-178.

24. Lim SS, Dandona L, Hoisington JA, et al. India's Janani Suraksha Yojana, a conditional cash transfer programme to increase births in health facilities:an impact evaluation. The Lancet. 2010;375(9730):2009-2023.

25. Paria B, Bhattacharyya A, Das S. A comparative study on menstrual hygiene among urban and rural adolescent girls of West Bengal. Journal of family medicine and primary care. 2014;3(4):413. 